\title{
Laboreal
}

Volume $6 \mathrm{~N}^{\circ} 2$ | 2010

Varia

\section{Pan y veneno : reflexiones para una investigación sobre el 'ambientalismo del trabajo' en Italia, 1968 - 1998}

Pão e veneno : reflexões para uma investigação sobre o 'ambientalismo do trabalho' em Itália, 1968-1998

Pain et poison : quelques pistes pour une recherche sur "l'environnementalisme du travail "en Italie, 1968-1998

Bread and poison : reflections for a research on labour environmentalism in Italy, 1968-1998

\section{Stefania Barca}

\section{(2) OpenEdition}

\section{Journals}

Edición electrónica

URL: http://journals.openedition.org/laboreal/8692

DOI: 10.4000/laboreal.8692

ISSN: 1646-5237

Editor

Universidade do Porto

Referencia electrónica

Stefania Barca, «Pan y veneno : reflexiones para una investigación sobre el 'ambientalismo del trabajo' en Italia, 1968 - 1998 », Laboreal [En línea], Volume 6 No2 | 2010, Publicado el 01 diciembre 2010, consultado el 10 octubre 2019. URL : http://journals.openedition.org/laboreal/8692 ; DOI : 10.4000/laboreal.8692

Este documento fue generado automáticamente el 10 octubre 2019

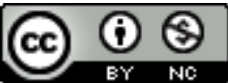

Laboreal está licenciado com uma Licença Creative Commons - Atribuição-NãoComercial 4.0 Internacional. 


\section{Pan y veneno : reflexiones para una investigación sobre el 'ambientalismo del trabajo' en Italia, 1968 - 1998}

Pão e veneno : reflexões para uma investigação sobre o 'ambientalismo do trabalho' em Itália, 1968-1998

Pain et poison : quelques pistes pour une recherche sur "l'environnementalisme du travail " en Italie, 1968-1998

Bread and poison : reflections for a research on labour environmentalism in Italy, 1968-1998

Stefania Barca

\section{NOTA DEL EDITOR}

Manuscrito recibido en : septiembre/2010

Aceptado tras peritaje : noviembre/2010

\section{Premisa}

1 La reflexión sobre el ambientalismo del trabajo en Italia se presentó inicialmente de forma problemática. Las luchas sindicales emprendidas al inicio de los años 60 , luchas que se llamaron 'contra la nocividad', o sea, donde los trabajadores querían tener el control sobre las condiciones ambientales en su fábrica, acabaron manteniendo una débil y a veces conflictiva relación con las batallas ambientalistas consideradas de élite (por ejemplo, contra la especulación inmobiliaria o para el establecimiento de áreas protegidas). En el recuerdo de uno de los protagonistas de esa época, el médico del 
trabajo Massimo Menegozzo, la oportunidad para vincular las luchas contra la nocividad en las fábricas con la lucha más general contra la contaminación industrial no fue recogida por el movimiento sindical [1]. Sin embargo, una simple revisión de la documentación que se encuentra disponible sobre el tema, así como de la literatura historiográfica producida hasta el momento, deja claro que al considerar las luchas contra la nocividad apenas como parte de la lucha sindical italiana de aquellos años o como parte del desarrollo de la medicina del trabajo - temas que habían sido centrales y bien analizados hasta el momento - se estaba subestimando el valor cultural y político de estas mismas luchas, y la contribución que representaron para el desarrollo del movimiento ecológico italiano.

2 La historia que se encuentra en este artículo comenzó por el encuentro entre un grupo de 'especialistas' en higiene laboral y un grupo de obreros y obreras, que tuvo lugar en el corazón del triángulo industrial italiano entre los años 60 y los 70. Este encuentro condujo a nuevas formas de conciencia ambiental y de acción política que voy a designar como "ambientalismo del trabajo". A continuación, la narración se moverá hacia un área de la industria petroquímica al sur de Italia entre los años 70 y los 90, donde las contradicciones de la relación entre el trabajo, la salud y el medio ambiente, explotaban y subían de diferentes formas. Mi intención, con el presente artículo, es sugerir una nueva visión sobre estas luchas, inspirados en una literatura históricoambiental recientemente hecha sobre la nocividad industrial [2]. .El punto de partida teórico fue la unidad orgánica y material entre el trabajo, el ambiente y la salud que los obreros y las obreras y sus familias experimentan en sus cuerpos según sus condiciones de trabajo y de vida [3]. Este artículo también tiene como objetivo realzar que la unidad orgánica entre los seres humanos y la naturaleza a través del trabajo ha sido ofuscada por las fuerzas políticas dominantes, así como por los contrastes entre el movimiento obrero y el movimiento ecologista. La historia del movimiento ecologista italiano, mientras tanto, permite mostrar la posibilidad para la existencia de una conciencia antagonista sobre el riesgo laboral, que surgió en algunos momentos y lugares a lo largo del último medio Siglo, y cómo ese conocimiento ha sido capaz, de varias maneras, de desafiar las formas conocidas del conocimiento científico e, incluso, del poder político-económico.

3 Por último, este artículo pretende contribuir en la construcción de una historia para un trabajo ambientalmente consciente. Nuestros argumentos se basan en la idea de que los trabajadores y las trabajadoras, y sus familias, no sólo llevan la marca del desarrollo capitalista en sus cuerpos, sino que fueron los que pagaron el precio último durante la transformación ambiental tumultuosa del periodo de la post-guerra $\mathrm{y}$, por estas razones, la historia del trabajo incluye una parte importante de la historia del movimiento ecológico en Italia. La formación de una conciencia ecológica del trabajo con base en la manifestación del daño corporal, fue perjudicada por la gran mistificación cultural y política que afectó al crecimiento económico italiano. Actualmente es responsabilidad de la obra historiográfica traer a la luz no solamente los aspectos ambientales de las luchas de los trabajadores por su salud, sino conseguir el accionar de fuerzas contrarias a la formación de un frente único de conciencias de clases y conciencias ecológicas.

El presente artículo es el resultado de la reorganización de un trabajo presentado en la Conferencia 'Dangerous Trade. Histories of industrial hazards across a globalizing world', realizada en la Universidad de Stony Brook (EUA), en Diciembre del 2007. 
Constituye un levantamiento preliminar de las temáticas y cuestiones que se relacionan con el tema del movimiento ecológico del trabajo en Italia, utilizando para ello una variedad de fuentes : 1 . Artículos de la Revista Sapere, publicados durante el periodo de dirección de Giulio Maccacaro ; 2. Informes de los Servicios Médicos para el Ambiente de Trabajo de la Provincia de Milán (1972-1976), 3. Entrevistas (realizadas por la autora), con dos de los protagonistas de las luchas contra la nocividad; 4. Entrevistas (publicadas) sobre el caso de la industria petroquímica en Manfredonia, y 5. Literatura histórica sobre la nocividad industrial y sobre el contexto económico y político del periodo considerado.

\section{Los costos sociales del 'Boom Económico' (1958-1969)}

Entre 1955 y 1970, cerca de cuatro millones de personas emigraron de las áreas rurales del sur de Italia para el triángulo industrial, la mayoría de ellos en busca de trabajo en las fábricas, y muchas lo consiguieron en las áreas de crecimiento rápido : el acero, la mecánica y la petroquímica. Desde finales de los años 60 el Estado inició una política de localización industrial al sur del país que transformó radicalmente a las zonas costeras con la creación de grandes fábricas, la mayoría de ellas de propiedad estatal, especialmente en el sector petroquímico [4].

6 Como resultado de esos cambios, el país sufrió la transición epidemiológica típica de las economías industrializadas, o sea, fue desde la prevalencia de enfermedades infecciones a la prevalencia de enfermedades degenerativas (como el cáncer y las enfermedades cardiovasculares, especialmente aquellas que se relacionaban con la contaminación ambiental por hidrocarburos, mercurio y benceno. El INAIL (Instituto Italiano para la Prevención Social) financió la investigación en el campo de la higiene laboral, aunque esta fue esencialmente dirigida hacia el seguro sobre riesgos y patologías clínicas, en vez de hacia la prevención. Como acuerdo tácito se estableció que la salud (y no sólo la de los trabajadores) era el precio que se tenía que pagar para el desarrollo industrial del país. Frente al impresionante deterioro de las condiciones de trabajo en las fábricas, ligado a la intensificación de las tasas de producción, los sindicatos comenzaron a adoptar, por su parte, estrategias defensivas focalizadas en la máxima obtención de compensaciones debido a los accidentes y algunas enfermedades de trabajo (muy difíciles de documentar y siempre sujetas a rechazo por parte de las autoridades sanitarias). Las compensaciones o - como hemos llegado a decir - las "monetarizaciones" del riesgo laboral, no hacían otra cosa que impedir su prevención, por el simple hecho de que el INAIL cubría los gastos (quitando, seguidamente, gran parte de ellos del presupuesto público), dejando a los dueños de las empresas libres de cualquier responsabilidad criminal y civil relacionada con los riesgos laborales. El "milagro" de la economía italiana se produjo en gran parte debido a los bajos costos de la salud de los trabajadores y a las coberturas de los seguros provistos por el Estado [5].

El movimiento ecológico del trabajo en Italia comenzó cuando un grupo de "especialistas" militantes - médicos, ingenieros y sociólogos del trabajo - comenzaron a entrar en las fábricas del milagro económico, solicitados por el movimiento sindical, durante la etapa crucial del conocido como "otoño caliente"; conociéndose, así, los riesgos a que eran sometidos los trabajadores y las trabajadoras. Este primer encuentro tenía por un lado las reflexiones sobre las relaciones entre saber y poder, típicas del 
movimiento estudiantil, y, por otro, la conciencia del movimiento obrero (y de la opinión pública) sobre la existencia de un enorme problema de salud y seguridad del trabajo causado por el llamado "milagro económico".

8 Esta conciencia fue la que también condujo a un amargo debate, después de que un nuevo Estatuto de los Trabajadores fuese aprobado por el Parlamento Italiano (en la Ley 300 de 1970). El Estatuto incluía dos Artículos que expresamente reconocían el derecho de la clase trabajadora para ejercer un control de forma directa (o sea, sin que mediasen los inspectores del trabajo o los "especialistas" en el mandato del pago) sobre las condiciones ambientales de la fábrica. Esto se puso en práctica durante los años 60 en algunas instituciones pioneras, pero después de 1970 este control sobre las condiciones del trabajo se extendió exitosamente sobre todo en las grandes fábricas del triángulo industrial. Esto permitió la producción de una significativa contribución intelectual, publicadas en Revistas como "Medicina dei Lavoratori" y " Medicina Democrática", en Revistas no especializadas como "Sapere" (particularmente durante el período en que fue dirigida por Giulio Maccacaro, que también dirigía simultáneamente la serie "Medicina e Potere" de la Editorial Feltrinelli), y en una extensa literatura especializada [ $\left.{ }^{6}\right]$.

Encontramos en la reconstrucción que hacemos de la historia y en los testimonios de las personas que estuvieron implicadas, cómo la lucha por la salud en las fábricas se utilizó para preparar las condiciones para la Reforma General del Sistema Nacional de Salud Pública, que fue finalmente aprobada en 1978, después de cinco huelgas generales con "sangre y lágrimas" [7]. Estos fueron años en los que las fábricas eran consideradas foco de la política y la vida social italiana, en las que la unión entre salud pública y salud del trabajo formaban parte de la manera común de entender la realidad social y sanitaria del país. La Reforma de Salud Nacional apareció, por estos motivos, como una victoria de la alianza entre la ciencia militante y el conocimiento popular, entre especialistas/activistas y la clase trabajadora; lo que trajo como consecuencia una Reforma mucho más amplia y general, incluyendo a toda la sociedad del país. Como vamos a ver en la segunda parte del presente artículo, el impacto de estas reformas institucionales sobre los órganos de trabajadores y trabajadoras, y sobre las condiciones ambientales de la vida y el trabajo en el país, fue fuertemente influenciado por las jerarquías del contexto geográfico italiano y por los cambios políticos y económicos de las siguientes décadas.

\section{El ambiente de trabajo y la medicina militante : la experiencia de los SMAL}

10 El abordaje de los Sindicatos relacionado con la salud en el trabajo fue radicalmente revisado durante los años 60 , después de una revolución que se produjo en materia de higiene laboral que proponía la utilización, de forma innovadora, de la formación de conocimientos sobre las condiciones de salud en el ambiente de trabajo. Esta nueva forma de conocimiento participativo estaba basada en una revisión radical del papel de los "especialistas" en higiene laboral, desde los científicos imparciales pero que de hecho se encontraban al servicio de las empresas, hasta los "intelectuales orgánicos" al servicio de la clase trabajadora. Brevemente, la medicina laboral se volvió una ciencia militante. Esta militancia se basó en dos elementos principales. El primero de ellos fue la formulación de forma simple y clara de los principios básicos de higiene industrial, 
considerados como los 4 principales factores de riesgo: 1) los factores inespecíficos (como el ruido, el microclima, las vibraciones, las radiaciones, etc.), 2) los factores específicos del ambiente laboral (la exposición a sustancias tóxicas o explosivas), 3) los factores relacionados al esfuerzo físico (el esfuerzo, la postura y los movimientos incómodos), y 4) los factores relacionados con la organización del trabajo (la monotonía, el ritmo excesivo, la ansiedad, la responsabilidad). El segundo elemento de esta nueva medicina del trabajo consistió en la propuesta del principio de "non delega" en materia de salud en los locales de trabajo, o sea, que el impacto del trabajo sobre la salud tenía que definirse directamente por la clase trabajadora y no por los "especialistas". Esto llevó a la formulación de nuevos instrumentos de conocimiento administrados por los trabajadores y los sindicatos, entre ellos un cuestionario para la recogida de datos, registros de datos ambientales y bioestadísticos, y registros de datos sobre el estado de salud de los trabajadores [8]. Durante esta época, el tema del "ambiente laboral" ocupó un lugar destacado en el debate político interno del movimiento izquierdista. Se creó un taller de forma permanente, renombrado como "el club del ambiente", dentro del CGIL - el principal sindicato italiano -, con la misión de formular y probar nuevas formas de conocimiento/acción contra la nocividad [ $\left.{ }^{9}\right]$.

11 Una experiencia de gran importancia dentro de este fervor creativo relacionado con el tema de los riesgos laborales, fue la que tuvo los SMAL - Servicios de Medicina para los Ambientes de Trabajo, una estructura creada por iniciativa del CGIL-CISL-UIL (la Federación de los tres sindicatos nacionales más grandes), e instituida por el Gobierno Regional de Lombardía a finales de 1972, con la tarea de tornar operacional de forma inmediata el derecho de control de los trabajadores en el ambiente de las fábricas. La experiencia de los SMAL se marcó fuertemente por el contexto regional, así como por el contexto histórico : se crearon en el corazón del área industrial (en la Provincia de Milán) y en la cumbre de la era dorada de la lucha por la salud en las fábricas (1972-1976). Tuvieron el valor de ser una experiencia pionera, que tuvo la responsabilidad histórica de mostrar la forma en que se podía configurar el ambiente laboral y los resultados de este proceso.

Los SMAL cumplieron con su tarea haciendo que los trabajadores y las trabajadoras participaran activamente, pasando, de acuerdo con el "propósito" de la investigación, a actores. Fueron los miembros del 'Consejo de la Fábrica' los que llamaron a los "especialistas" para cualquier intervención, y todas las acciones que se tomaron en el ámbito de la investigación fueron decididas por una Comisión de "salud y seguridad" que formó el mismo Consejo. La intervención de los SMAL se basó, esencialmente, en crear herramientas para un estudio auto-dirigido por parte de los trabajadores, lo que permitió a cada trabajador y trabajadora que hiciesen las mediciones relacionadas con los cuatro grupos de riesgo, así como con su propia condición psico-física y con cualquier situación de malestar que se pudo haber presentado. A partir de los datos ambientales recogidos por los trabajadores, los SMAL tuvieron la oportunidad de hacer un estudio nosológico y, eventualmente, exigir la gestión de medidas preventivas y/o de realizar cambios reales en la estructura organizacional y técnica de la Empresa.

En resumen, los SMAL tuvieron el poder de transformar la percepción de los trabajadores y de las trabajadoras sobre los riesgos presentes en el local de trabajo, y de realizar cambios importantes en las formas de organización de la producción con el objetivo de evitar el daño en vez de compensar lo que ya pudo haber sucedido. Movilizar el conocimiento del riesgo que la clase trabajadora podría sufrir sobre sus 
cuerpos, y que pudiera ser utilizado en primera persona para realizar cambios en las condiciones de trabajo de la fábrica, constituyó la principal finalidad del surgimiento de los SMAL. "La visión que un profesional puede tener sobre los peligros de un ambiente de trabajo - de acuerdo con un informe de los SMAL - puede llegar a ser lo bastante suficiente y parcial si no se confronta con la opinión de quien vive ahí durante ocho horas al día". En resumen, el ambiente de trabajo comenzó a verse como un tipo específico de ecosistema, y la clase trabajadora era quien mejor lo conocía $\left.{ }^{10}\right]$.

Los SMAL no funcionaban como un organismo científico fuera del mundo laboral con el objetivo de extraer conocimiento a partir de los trabajadores. Estaban, en cambio, constituidos por un grupo de científicos/activistas al servicio de la clase trabajadora bajo la indudable hegemonía de los Sindicatos. Fueron los propios Sindicatos los que presionaron para la creación de los SMAL a nivel de Provincias y Regiones, después de haber tenido el presupuesto por parte de un número de Gobiernos locales; fueron los Sindicatos los que organizaron los cursos de formación para aspirantes médicos de los SMAL y actuaron como intermediarios entre ellos y las organizaciones de trabajadores ; fueron los Sindicatos los que coordinaron los esfuerzos a nivel Regional. La naturaleza militante de los SMAL y, por tanto, la prioridad política relacionada con la defensa e los trabajadores contra los riesgos, se hizo evidente en los informes relacionados con la actividad realizada en los años 1972-1976 en la Provincia de Milán. El lenguaje usado en los textos reveló cómo los médicos [11] de los SMAL eran mucho más que "especialistas" de la salud en la realización de un servicio público : eran parte de una batalla cultural más general contra el conocimiento/poder y en función de la subjetividad de la clase trabajadora, usando para ello al ambiente de trabajo como un espacio importante de lucha política y social. Se dio con frecuencia la situación donde su acción implicó cuestiones que sobrepasaban el ambiente en las fábricas, incluyendo la relación que se producía entre esta y su entorno, donde se mantenían características políticamente muy delicadas, con conflictos entre el trabajo y el medio ambiente.

En Cinisello Balsamo, por ejemplo, surgió un complejo debate en torno al ruido proveniente de la fábrica de acero "Terzago", que colocó a los trabajadores en conflicto con la población local. La Dirección respondió a las protestas de la población, ordenando el cierre de las ventanas, aumentando de esta forma la carga de ruido perjudicando a los trabajadores y trabajadoras, amenazando con el cierre de la fábrica en caso de que hubiese reclamaciones por parte de ellos. Terzago era una pequeña empresa con una débil presencia de los sindicatos, donde la intervención del SMAL se produjo por un llamado del Comité Anti-ruido local. Por este motivo, fue muy probable que los trabajadores respondieron de forma negativa a la presencia del SMAL, sintiendo que era una amenaza. Esta situación fue políticamente sensible y exigió la capacidad de superar la aparente contradicción de los trabajadores con los ciudadanos en relación al tema de la salud, así como también exigió la capacidad para unir estos dos factores en la lucha contra la contaminación. El SMAL de la localidad definió el problema del ruido como un problema, en primer lugar, de salud laboral y actuó de forma que pudiese unir los dos factores implicados desde el primer momento. Esta línea de acción necesitó de una forma de intervención que no fuese estrictamente profesional, pues en un primer momento se reunió a los trabajadores y trabajadoras con la población local a través de asambleas de barrio, junto con la participación de Especialistas en Otorrinolaringología de la Universidad de Milán y representantes el Gobierno local. Fue sólo después que los SMAL comenzaron con las intervenciones en las condiciones de trabajo y con el levantamiento audiométrico. Los resultados evidenciaron una sordera parcial crónica 
en el $30 \%$ de los trabajadores examinados y un choque acústico en el $36 \%$, la mayoría mujeres. Sin embargo, la intervención del SMAL no se limitó a la verificación de daños para la salud de los trabajadores, sino que comenzó a buscar una solución técnica. Mediante la cooperación de "ingenieros democráticos", según se encuentra descrito en el informe, se presentaron propuestas concretas para el control de las vibraciones y la transmisión de ondas acústicas en el local de trabajo. Al final, la Dirección no solo no cuestionó los resultados de la investigación, sino que se mostró dispuesta en adoptar todas las soluciones propuestas [ $\left.{ }^{12}\right]$.

La relación entre los conflictos ambientales y el área laboral se evidenció en otro informe sobre la Empresa "Metal Lámina" en Assago. En este caso, fue el Consejo de trabajadores de una empresa cercana que llamó la atención del Servicio Municipal de Ecología sobre las emisiones de "Metal Lámina", los que, a su vez, buscaron la intervención del SMAL. La investigación evidenció que el ambiente de trabajo estaba contaminado con polvo de plomo en cantidades diez veces superiores a las permitidas. Los trabajadores contaron que cinco perros guardianes habían muerto dentro de la fábrica el pasado año, probablemente debido a la ingestión de polvo depositado sobre el suelo. El SMAL dio las instrucciones para la admisión en hospitales de ocho de los trece fundadores de la fábrica y ordenó parar la producción de todo el Bloque de Fundición. La administración de la Empresa amenazó con cerrar la fábrica, pero al final, no solo decidió aplicar todas las medidas relacionadas con la reducción de humo y polvo propuestas por el SMAL y por el Servicio Municipal de Ecología, incluyendo la instalación de un purificador de agua, sino que sugirió que el SMAL fue consultor de la Empresa en cuestiones de salud y medio ambiente [13].

El comportamiento de la "Metal Lámina" parece que fue bastante común en otras pequeñas y medianas empresas donde se produjo la intervención de los SMAL. El único caso donde se encontró resistencia, y hasta oposición abierta, fueron las empresas del Grupo Montedison, donde la Administración perjudicó por todas las vías la admisión de especialistas de los SMAL, alegando tener su propio servicio médico [ ${ }^{14}$ ]. Este hecho introduce el tema de la próxima sección, relacionado con las luchas contra la nocividad en las grandes empresas petroquímicas estatales.

\section{Manfredonia 1976 - 1998 : ambiente y trabajo en un polo petroquímico del sur}

18 En la mañana del 26 de Septiembre de 1976, apenas dos meses después de accidente en Séveso, la explosión de una columna de arsénico en la Planta ANIC de Manfredonia, en la Provincia de Foggia, causó la descarga en la atmósfera de una nube de 32 toneladas de dióxido de arsénico en polvo, que cayó sobre la ciudad y se depositó en terrenos agrícolas de los alrededores [15]. Los primeros síntomas de envenenamiento en masa fueron evidentes después de la muerte de muchos animales y la hospitalización de cerca de una centena de personas en pocas horas. Los pacientes fueron casi todos trabajadores de la fábrica y habitantes del barrio Monticchio, un conglomerado de viviendas sociales construidas cerca de la Plata ANIC. Increíblemente, la Dirección de la plata negó la existencia de cualquier riesgo epidemiológico e hizo que las personas fueran a trabajar de forma regular a partir del lunes siguiente. Como medida preventiva, un equipo formado por el personal de mantenimiento fue enviado a limpiar el interior del establecimiento, sin cualquier protección, apenas con sus escobas. Estos 
trabajadores estuvieron toda la noche recogiendo el polvo de arsénico depositado, para que la Planta volviese a funcionar 'normalmente' al día siguiente.

El accidente aconteció en un área sureña que sufrió una elevada tasa de desempleo crónico y emigración, un área cuyos recursos más importantes eran los terrenos agrícolas, usado sobre todo en la plantación de oliveras ; la pesca y el paisaje natural/ histórico (Manfredonia es la entrada para la península de Gargano). Estos recursos se vieron seriamente comprometidos no sólo por el accidente de 1976, sino debido a los más de 20 años de actividad del establecimiento ANIC (que posteriormente recibió el nombre de Enichem). En 1988, la Organización Mundial de la Salud clasificó el área como de "alto riesgo de crisis ecológica" por causa de la contaminación de la cadena alimentaria, y sugirió la creación de un observatorio epidemiológico de forma permanente [ $\left.{ }^{16}\right]$. Sin embargo, la población continuó ignorando las amenazas relacionadas con el período de actividad de la industria petroquímica en el territorio y con los efectos de la propagación de la contaminación por arsénico que sucedió en 1976. La prioridad política (y, por supuesto, la de la empresa) fue evitar de inmediato la diseminación de información para prevenir una situación semejante a lo que aconteció en Séveso, donde hubo una movilización de ciudadanos, partidos políticos, organizaciones sociales, el bloque de la Planta, evacuaciones, participación de especialistas extranjeros y una alerta de la opinión pública internacional. Nada de esto se produjo en Manfredonia, excepto por algunas voces, como la de la Democracia Proletaria, el único Partido que organizó una manifestación anti-ANIC importante, y a través del que los trabajadores, pescadores y habitantes del barrio Monticchio denunciaron los ataques sobre el medio ambiente y la salud que hizo la Empresa con el consentimiento tácito de las autoridades locales [ $\left.{ }^{17}\right]$.

En Manfredonia no hubo intervención de cualquier SMAL, ni en esa vez ni en ninguna otra durante más de 20 años de operaciones de la Planta Enichem. El único médico serio que fue visto por la compañía, según uno de los trabajadores entrevistados por Giulio di Luzio (2003) para su libro-reportaje Los Fantasmas de Enichem, fue despedido dos meses después. Por el contrario, el incidente de 1976 reveló la existencia de un patrón de relaciones bien establecidos entre la medicina y el poder político, con el objetivo de salvaguardar los intereses de la industria petroquímica, en detrimento de los trabajadores y de la población de Manfredonia. El suministro de datos de laboratorio de forma extremadamente tardía y la manipulación de forma deliberada de información relacionada con los niveles de contaminación permitidos (MAC), fueron las herramientas que usaron los médicos de las Clínicas del Trabajo de Bari y de Milán, algunos de los cuales eran consultores de ANIC, para colaborar con las autoridades y con la Empresa en función de mantener en secreto los efectos reales del accidente.

21 La falta de iniciativa de los Sindicatos (principalmente dirigidos por hombres), tuvo un efecto inesperado en la emergencia de otra forma de agencia política : la eco-feminista [18]. Fue realmente un grupo de 40 mujeres, en gran parte moradoras del barrio Monticchio, que en conjunto con la organización Bianca Lancia - Movimiento Ciudadano de Mujeres, se movilizaron para hacer un reconocimiento del daño. El Movimiento ganó un importante avance en 1978, cuando el tribunal de Justicia Europeo acordó evaluar el caso, aunque el veredicto final, que salió diez años después, se mostró muy por debajo de las expectativas. Las mujeres de Manfredonia consiguieron el reconocimiento sólo por los daños materiales, esto quiere decir, por la violación de su "derecho de conocer" los riesgos potenciales que existían en el establecimiento. Pero 
no consiguieron lo que ellas esperaban, es decir, el reconocimiento de los daños biológicos : la contaminación del aire, del agua, del suelo, de los cuerpos humanos $\mathrm{y}$, por tanto, de todo el ambiente en Manfredonia. En resumen, la Empresa fue condenada por esconder información sobre el riesgo, pero no por su responsabilidad directa de causar el daño.

En los 20 años que siguieron al accidente de 1976, la relación entre la clase trabajadora y la Ciudad de Manfredonia fue compleja y llena de contradicciones, a veces manifestándose a través de conflictos abiertos. Aún así, un evento significativo se produjo en Manfredonia, abriendo una nueva oportunidad histórica a el ambientalismo de la clase trabajadora, y una vez más fue a través de un encuentro : entre el trabajador retirado Nicola Lovecchio y el médico radiólogo Maurizio Portulari, que tuvo lugar en el Hospital de San Giovanni Rotondo en 1994 [19]. En este momento, la historia de Manfredonia se encuentra con la de los trabajadores del grupo Enichem en el área de Porto Marghera. Antes del encuentro con Lovecchio, de hecho, Portulari había leído el dossier elaborado por el ex-trabajador de Marghera, Gabriele Bortolozzo, y publicado por la revista Medicina Democrática, en 1994 [ ${ }^{20}$ ]. Portulari le propuso a Lovecchio, exgerente del Departamento de Empacado de Fertilizantes de Enichem, comenzar a recoger informaciones sobre su enfermedad y sobre las de sus colegas, y sobre las condiciones ambientales en la fábrica. Lovecchio aceptó (manteniendo su compromiso hasta su muerte) y de esta forma comenzó una investigación/acción colectiva que incluyó a decenas de colegas y a sus familias, resultando un proceso judicial que todavía se mantiene activo, una acción civil de gran importancia para las centenas de personas involucradas en el proceso, así como para el posible desarrollo del movimiento ecologista italiano.

\section{Conclusiones}

Los hechos de los SMAL de Lombardía, y de Anic/Enichem de Manfredonia, indican perspectivas diferentes para la investigación histórica y etnográfica sobre el ambientalismo italiano y sus diferentes manifestaciones. Este artículo visa resaltar los principales elementos que manifiestan una continuidad entre las luchas contra la nocividad durante los años 1970-78, y la nueva temporada, que comenzó en los años 90, con el proceso de Marghera. Este proceso abrió un nuevo capítulo en la historia de las luchas contra la nocividad, un capítulo en que la acción del Sindicato fue substituida por (e a veces entró en conflicto con) la acción del individuo ; aunque hubo momentos importantes en la continuidad con el periodo anterior de luchas. Si el Estatuto de los Trabajadores parece hoy en día una conquista lejana sin potencial revolucionario, la cultura de la medicina militante se mantuvo viva, y el papel de los expertos/activistas (no sólo los médicos, sino también los ingenieros y abogados) para apoyar las quejas de los trabajadores, se mantuvo fundamental en el desarrollo de investigaciones judiciales que hubo como consecuencia tanto en Manfredonia como en Marghera. Igualmente de gran importancia fue, dando continuidad al proceso de los años 70, la contribución hecha por el feminismo en la organización de el ambientalismo de los ciudadanos y en la batalla para que fuesen reconocidos los daños. El papel de la subjetividad femenina y, por supuesto, el elemento de la acción legal, acercó la historia en Manfredonia con la de los movimientos para la 'justicia ambiental', como forma de lucha contra la contaminación que nació en los EUA a mediados de los años 80 , y que se expandió 
globalmente [ ${ }^{21}$. La subjetividad de la clase trabajadora, organizada o no, en la lucha contra los efectos nocivos, fue un elemento común en las luchas por la 'justicia ambiental' en otros contextos geográficos e históricos, así como lo fueron también sus contradicciones y dificultades. Este, sin embargo, continúa siendo el elemento menos estudiado del llamado "ambientalismo popular" y constituye el aspecto donde futuras investigaciones pueden tener resultados más interesantes.

\section{BIBLIOGRAFÍA}

Adorno, S. y Neri Serneri, S. (Orgs.) (2009). Industria, ambiente e territorio. Per una storia ambientale delle aree industriali italiane. Bologna : Il Mulino

Allen, B. (2003). Uneasy Alchemy. Citizens and Experts in Louisiana's Chemical Corridor Disputes, Harvard: MIT Press: Harvard

Allen, B. (2007). La trasformazione industriale del basso corso del Mississippi in Louisiana, I Frutti di Demetra. Bollettino di Storia e Ambiente, 14, 47-56.

Armiero, M. (2009). Un altro ambientalismo è possibile. Anzi c'è già. In Joan Martinez Alier, Ecologia dei poveri (xi-xvii.). Milano : Jaca Book

Barca, S. (org) (2005). Massimo Menegozzo : Lavoro e salute in Italia. I Frutti di Demetra.

Bollettino di Storia e Ambiente, 5, 63-70

Berlinguer, G. (1991). Storia e Politica della Salute. Milano : Franco Angeli

Bettin, G. (org) (1998). Petrolkimico. Le voci e le storie di un crimine di pace. Milano: Baldini Castoldi Dalai

Calavita, K. (1986). Worker Safety, Law and Social Change: The Italian Case, Law \& Society Review $2,189-228$

Carnevale, F. y Baldasseroni, A. (1999). Mal da Lavoro : Storia della Salute dei Lavoratori. RomaBari : Laterza

Centemeri, L. (2006). Ritorno a Seveso. Il Danno Ambientale, il suo Riconoscimento, la sua Riparazione. Milano : Mondadori

CGIL-CISL-UIL Federazione Provinciale di Milano (1976). Salute e Ambiente di Lavoro. L'esperienza degli SMAL. Milano : Mazzotta.

Crepas, N. (1998). Industria. In Massimo Firpo, Nicola Tranfaglia e Pier Giorgio Zunino (Orgs), Guida all'Italia Contemporanea, 1861-1997 (287-422). Milano : Garzanti

De Luna, G. (2008) Le ragioni di un decennio. 1969-1979. Militanza, violenza, sconfitta, memoria. Milano: Feltrinelli

Dewey, S., (1998). Working for the environment. Organized Labor and the origins of Environmentalism in the United States, 1948-1970, Environmental History, 1, 45-63

Di Luzio, G. (2003). I Fantasmi dell'Enichem. Milano : Baldini Castoldi Dalai 
Firpo, M. (2009). Eixos de desenvolvimento econômico e geração de conflitos socioambientais no Brasil : desafios para a sustentabilidade e a justiça ambiental, Ciência e Saúde Colectiva, 6, 1983-94.

Gordon, S., (1998). Shell No! OCAW and the Labor-Environmental Alliance, Environmental History, 4, 460-87

Graf von Hardenberg, W. \& Pelizzari, P. (2008). The environmental question, employment and development in Italy's left, Left History 2, 77

Langiu, A. y Portaluri, M. (2008). Di Fabbrica si muore. San Cesario di Lecce : Manni

Luzzi, S. (2009). Il Virus del Benessere. Ambiente, Salute, Sviluppo nell'Italia Repubblicana. RomaBari : Laterza

Martínez Alier J. (2005). El Ecologismo de los Pobres. Conflictos Ambientales y Lenguajes de Valoración. Icaria : Barcelona.

Merchant, C. (2005). Radical Ecology. In Search for a Livable World. London : Routledge

Musso, S. (1998). Lavoro e occupazione. In Massimo Firpo, Nicola Tranfaglia e Pier Giorgio Zunino (Orgs), Guida all'Italia Contemporanea, 1861-1997 (485-544). Milano: Garzanti

Obach, B.K., (2004). Labour and the Environmental Movement. The Quest for Common Ground. Cambridge (MA) : The MIT Press

Oddone, I. y col. (1977). Ambiente di lavoro. La fabbrica nel territorio. Roma: Edizioni Scientifiche Italiane

Parsons, H.L (org) (1977). Marx and Engels on Ecology. Westport and London: Greenwood Press

Signorelli, A., (1995). Movimenti di popolazione e trasformazioni culturali. In Storia dell'Italia repubblicana, vol. 4. La Trasformazione dell'Italia : Sviluppo e Squilibri (pp. 589-658).Torino : Einaudi

Tomaiuolo, F. (2006). 1976-2006 : Trent'anni di arsenico all'Enichem di Manfredonia, I Frutti di Demetra. Bollettino di Storia e Ambiente, 12, 33-41.

Tonelli, P. (2006). Salute e lavoro. In Luigi Falossi (org), Il ‘900 : Alcune Istruzioni per l’Uso (pp. 45-65) Firenze : La Giuntina

Tonelli, P. (2007). La salute non si vende. Ambiente di lavoro e lotte di fabbrica tra anni '60 e '70. In Luigi Falossi and Francesco Loreto (orgs), I Due Bienni Rossi del ‘900. 1919-1920 e 1968-1969. Studi e Interpretazioni a Confronto (pp. 341-52). Roma: Ediesse

White, R., (1996). 'Are You an Environmentalist, or Do You Work for a Living?' Work and Nature. In William Cronon (org) Uncommon Grounds. Rethinking the Human Place in Nature,

(pp. 171-85). New York, W.W. Norton

\section{NOTAS}

1. Entrevista realizada por la autora. Ver Barca (org) (2005). Una visión diferente fue expresada, por ejemplo, por otro protagonista de la misma Estación, Ivar Oddone (1977). No existen muchos estudios históricos sobre la relación entre el movimiento ambientalista y el movimiento sindical en Italia. Las reconstrucciones de que disponemos, sin embargo, parecen apuntar a una dificultad para este tipo de relación, especialmente a partir de la recesión económica que se vivió de 1977 a 
1978. Para una revisión crítica sobre este tema ver, por ejemplo, en Graf von Hardenberg y Pelizzari 2008.

2. Sobre la relación entre el movimiento obrero y el movimiento ambiental en EUA, encontramos una historiografía especializada. Ver, por ejemplo, White (1995), Gordon (1998), Dewey (1998) y Obach (2004). Para la dialéctica entre los ciudadanos y los especialistas en las áreas de riesgo laboral, ver Allen $(2003,2007)$.

3. Esta mirada la encontramos en Karl Marx, especialmente en su Libro I de "El Capital", donde se abordan las condiciones de vida en las fábricas, relacionadas con la salud pública. También aparece dicho por Engels, en su famosa encuesta sobre las condiciones de la clase trabajadora inglesa. Una selección de textos de estos dos autores sobre el medio ambiente puede verse en Parsons (1977), pp. 185-205.

4. Relacionado con la industria y las migraciones internas en Italia consultar a Signorelli (1995), Musso (1998) y Crepas (1998). Relacionado con el impacto ambiental de la industrialización consultar a Adorno y Serneri (2009), y Luzzi (2009).

5. Ver en Carnavale y Baldasseroni (1999) 147-229; Calavita (1986); Berlinguer (1991).

6. Relacionado con la renovación de la higiene laboral italiana en los años 60 y 70, recomendamos ver en Carnevale y Baldasseroni (1999), 230-82. Ver también Tonelli (2006, 2007). Sobre los movimientos político-juveniles de los años 70 en Italia, ver en De Luna (2008).

7. La expresión fue relatada por Massimo Menegozzo: ver en Barca (org) (2005), 67. En la misma entrevista, se destacan las relaciones entre las luchas contra la nocividad y la Reforma de Salud Pública, asunto que también encontramos en CGIL-CISL-UIL (1976) 189-99.

8. Esta renovación de la medicina del trabajo en Italia, en los años 60-70, hacía que fuese considerada el tipo de ciencia que la filósofa Sandra Harding llamó como 'strong objectivity', o sea, como un conocimiento construido de forma compartida entre los especialistas/activistas y los sujetos sociales oprimidos por el saber/poder dominante. En la forma de 'strong objectivity', la objetividad científica no se manifiesta por la imparcialidad del sujeto conocedor, sino por su habilidad de reconocer las relaciones de poder que encontramos en el proceso de conocimiento. Una discusión sobre las potencialidades de la 'objetividad fuerte' en el estudio de los riesgos laborales podemos encontrarlas en Allen (2003), pp. 6-7, 118, 140, 149.

9. En el relato que cuenta Massimo Menegozzo, la individualización de los cuatro factores de riesgo se realizó por un grupo de sociólogos del trabajo de la Universidad de Torino en la segunda mitad de los años 60. Al respecto consultar en Barca (org) (2005), 65. Esto fue dado a conocer a partir de 1969 por una publicación sindical titulada L'ambiente di lavoro, escrita por Gastone Marri e Ivar Oddone.

10. Ver CGIL-CISL-UIL (1976), 110.

11. En el texto referido no aparecen nombre de mujeres, por tanto, podemos considerar que al menos en esta fase los funcionarios SMAL fueron hombres.

12. El informe que recoge este caso aparece en el CGIL-CISL-UIL (1976), pp. 74-85.

13. Ibíd., 143-49.

14. Ibíd., 189.

15. En la historia del accidente en Manfredonia hago referencia al libro de Di Luzio (2003); también se puede consultar a Tomaiuolo (2006) y Luzzi (2009), pp. 152-55.

16. La información se encuentra en Di Luzio (2003), p. 74. El autor cita una parte de la publicación "Ambiente y Salud en Italia", donde la Organización Mundial de Salud declaró oportuno el establecimiento de un programa de vigilancia epidemiológica en la región de Manfredonia, para monitorizar los efectos del incidente del 76, a largo plazo, dada la latencia de los efectos para la salud, asociados a la exposición por arsénico.

17. Acerca del impacto social del desastre de Séveso ver en Centemeri (2006). Ver también en Luzzi (2009), pp. 140-155.

18. Sobre el eco-feminismo ver en Merchant (2005), pp. 193-222. 
19. El encuentro entre Portulari y Lovecchio fue el principal objetivo de la entrevista realizada por la autora con Portulari en Abril del 2009. Este encuentro también se encuentra narrando por Langiu y Portaluri (2008).

20. Ver en Bettin (org) (1998).

21. Sobre la justicia ambiental existe una amplia literatura en inglés, aunque algunos estudios están ahora disponibles en italiano, castellano y portugués. Por ejemplo, ver en Armiero (2009); Martinez Alier (2005); Firpo (2009).

\section{RESÚMENES}

Este artículo presenta una reflexión preliminar para la realización de una investigación histórica sobre un aspecto del movimiento ecologista italiano que hasta hoy ha sido poco considerado y que denomino como "ambientalismo del trabajo", para refirme a las luchas por la salud en los ambientes de trabajo y en la vida de la clase trabajadora. Mi análisis parte del encuentro entre un grupo de 'especialistas' en higiene laboral y un grupo de obreros y obreras, que tuvo lugar en el corazón del triángulo industrial italiano entre los años 60 y los 70. Este encuentro condujo a nuevas formas de conciencia ambiental y de acción política que designo como "ambientalismo del trabajo". A continuación, la narración se moverá hacia un área de la industria petroquímica al sur de Italia entre los años 70 y los 90, donde las contradicciones de la relación entre el trabajo, la salud y el medio ambiente, explotaban y remontaban de diferentes formas. Mi intención con el presente artículo es sugerir una nueva visión sobre estas luchas inspirada en la nueva literatura histórico-ambiental sobre la nocividad industrial.

Este artigo oferece uma reflexão preliminar para a investigação histórica sobre um aspecto até hoje pouco considerado do ambientalismo italiano: o que vou chamar "ambientalismo do trabalho", isto é as lutas pela saúde nos ambientes de trabalho e de vida da classe trabalhadora. A história começa desde um encontro - entre uma geração de 'especialistas' em higiene industrial e uma outra de trabalhadores/as de fábrica - que teve lugar no coração do triângulo industrial italiano entre os anos “ 60 e " 70 . Este encontro produziu novas formas de consciência ambiental e acção política, o que denominarei "ambientalismo do trabalho". Em seguida, a narração move-se para uma área de indústria petroquímica no Sul da Itália entre os anos '70 e ' 90 , onde as contradições da relação entre trabalho, saúde e meio ambiente explodiam e se reconfiguravam em diferentes formas. A minha intenção neste artigo é a de sugerir uma nova interpretação dessas lutas, inspirada por uma recente literatura histórico-ambiental sobre a nocividade industrial.

Cet article présente une réflexion préliminaire à une recherche historique sur un aspect peu exploré à ce jour de l'environnementalisme italien. Il s'agit de ce que je définis comme " environnementalisme du travail ", c'est à dire, les luttes pour la santé dans les environnements de travail et de vie, menées par la classe des travailleurs. L'histoire commence par une rencontre entre une génération d"' experts" en hygiène industrielle et une génération de travailleurs de l'industrie qui a eu lieu au cœur du "triangle industriel " italien entre les années 1960-1970. Cette rencontre a produit des formes nouvelles de conscience concernant l'environnement et l'action politique, ce que je nomme "environnementalisme du travail". Ensuite, la narration se déplace dans une région de l'industrie pétrolochimique au Sud de l'Italie entre les années 1970-1990. Ici 
les contradictions des relations entre travail, santé et environnement ont explosé et ont assumé différentes formes. L'objectif de cet article est d'avancer une nouvelle interprétation de ces luttes, à partir d'une littérature historico-environnementale récente centrée sur la nocivité industrielle.

This article presents a preliminary reflection for conducting historical research on an item which has so far been little considered in the study of the Italian environmental movement : what I will call "labour environmentalism", that is the struggle for health in the work and living environment of the working class. The story told in this article starts with the meeting between a group of 'specialists' in occupational hygiene and a group of workers, which took place in the heart of the Italian industrial triangle between 1960 and 1970. This meeting led to new forms of environmental awareness and political action that I will designate as "labor environmentalism". Then the story moves to an area of petrochemical industry in southern Italy between the 1970s and the 1990s, where the contradictions of the relationship between work, health and the environment exploded and were reconfigured in different ways. My intention with this article is to suggest a new vision of these struggles, inspired by a recent historical literature about industrial hazard.

\section{ÍNDICE}

Palabras claves: ambientalismo del trabajo, luchas contra la nocividad, "Servizi di Medicina per gli Ambienti di Lavoro", industria petroquímica, Manfredonia

Keywords: labour environmentalism, anti-toxic struggles, "Servizi di Medicina per gli Ambienti di Lavoro", petrochemicals, Manfredonia

Palavras-chave: ambientalismo do trabalho, lutas contra a nocividade, "Servizi di Medicina per gli Ambienti di Lavoro", industria petroquímica, Manfredonia

Mots-clés: environnementalisme du travail, luttes contre les nocivités, “Services de Médecine pour les Environnements de Travail ”, industrie pétrolochimique, Manfredonia

\section{AUTOR}

\section{STEFANIA BARCA}

Centro de Estudos Sociais, Universidade de Coimbra

sbarca@ces.uc.pt 